\title{
ALTERAÇÕES QUÍMICAS TEMPORAIS NAS FAIXAS DE ADUBAÇÃO E ENTRELINHAS DO POMAR, NUTRIÇÃO E PRODUÇÃO DE LARANJA APÓS CALAGEM SUPERFICIAL ${ }^{(1)}$
}

\author{
Jonez Fidalski ${ }^{(2)}$ \& Pedro Antonio Martins Auler ${ }^{(2)}$
}

\begin{abstract}
RESUMO
Apesar das diferenças químicas entre as faixas de adubação e entrelinhas de pomares de laranjeiras, o critério para a calagem superficial entre esses dois locais ainda é desconhecido. O objetivo deste estudo foi quantificar as alterações químicas temporais nas faixas de adubação e entrelinhas, nutrição e produção de laranja "Pêra" após calagens superficiais, em um Argissolo Vermelho distrófico latossólico, no noroeste do Paraná. Quatro calagens superficiais foram realizadas entre 1996 e 1999 para elevar a saturação por bases a $70 \%$, a partir da primeira colheita de frutos de laranja. Os calcários dolomítico e calcítico foram distribuídos sobre seis sistemas de manejo de plantas de cobertura permanente nas entrelinhas e nas faixas de adubação, as quais foram manejadas com roçadas mecânicas e herbicida pós-emergente, respectivamente. No período de 1996 a 2004 foram coletadas amostras de tecido foliar e do solo nas profundidades de 0-20 e 20-40 cm e avaliouse a produção de laranja. As máximas alterações químicas na camada de 0-20 cm de profundidade e no tecido foliar das laranjeiras (Ca) ocorreram quatro anos após sucessivas calagens superficiais anuais. A produção de laranja correlacionou-se negativamente com $\mathrm{Al}^{3+}$ e positivamente com $\mathrm{pH}-\mathrm{CaCl}_{2}, \mathrm{Ca}^{2+} \mathrm{e} \mathrm{Mg}^{2+}$ das faixas de adubação e do centro das entrelinhas. As calagens superficiais elevaram a saturação por bases na camada de 0-20 cm de profundidade até $46 \%$ nas faixas de adubação e $64 \%$ nas entrelinhas.
\end{abstract}

Termos de indexação: citros, acidez do solo, cálcio, saturação por bases.

\footnotetext{
(1) Recebido para publicação em maio de 2007 e aprovado em novembro de 2007.

(2) Pesquisador do Instituto Agronômico do Paraná - IAPAR. Área de Solos e Fitotecnia, Caixa Postal 564, CEP 87701-970 Paranavaí (PR). E-mails: fidalski@iapar.br, aulerpe@iapar.br
} 


\title{
SUMMARY: YIELD AND TEMPORAL CHEMICAL CHANGES IN FERTILIZER BANDS AND INTER-ROWS OF AN ORANGE ORCHARD AFTER SURFACE LIMING
}

\begin{abstract}
Despite the chemical differences in soils under the fertilizer bands and inter-rows in orange orchards, no criterion to determine the need of liming in the two areas is yet known. The objective of this study was to quantify the temporary chemical alterations in fertilizer bands and inter-rows of a "Pêra" orange orchard as well as nutrition and fruit yield after surface liming, in a Typic Paleudults, in northwestern Paraná, Brazil. The soil surface was limed four times between 1996 and 1999 to reach a base saturation of $70 \%$ since the first fruit harvest. Dolomitic and calcitic limestone were broadcast over six permanent groundcover plant types in the inter-rows and fertilization bands, which were mowed and sprayed with post-emergence herbicide. From 1996 to 2004 leaf samples, and soil samples in the layers $0-20$ and $20-40 \mathrm{~cm}$ were collected and the orange yield was evaluated. Maximal alterations of soil chemical characteristics and leaf nutrient concentrations (Ca) were observed after the fourth successive annual surface liming. The orange yield was negatively correlated with $\mathrm{Al}^{3+}$ and positively correlated with soil $\mathrm{pH}\left(\mathrm{CaCl}_{2}\right), \mathrm{Ca}^{2+}$ and $\mathrm{Mg}^{2+}$ in the fertilizer bands and inter-rows. Surface liming increased base saturation in the $0-20 \mathrm{~cm}$ layer to $46 \%$ in the fertilizer bands and to $64 \%$ inter-rows.
\end{abstract}

Index terms: citrus, soil acidity, calcium, base saturation, liming.

\section{INTRODUÇÃO}

A utilização de calcário na citricultura brasileira se deve à restrição química dos solos ácidos com a presença de $\mathrm{Al}^{3+}$ e baixa disponibilidade de $\mathrm{Ca}^{2+} \mathrm{e} \mathrm{Mg}^{2+}$ para as plantas cítricas. As laranjeiras caracterizamse pela maior absorção de Ca do que N (Mattos Jr. et al., 2003), sendo consideradas plantas calcífelas, com produção de frutos dependentes dos teores foliares de Ca (Fidalski \& Stenzel, 2006), motivo pelo qual, antes do plantio das mudas de laranjeiras, é recomendado o uso de calcário incorporado no momento do preparo do solo, de forma a garantir o crescimento radicular e a nutrição adequada das laranjeiras (Pavan \& Jacomino, 1998; Quaggio et al., 2005).

$\mathrm{O}$ método da determinação da necessidade de calagem para elevar a saturação por bases (V) a $70 \%$, que corresponderia ao $\mathrm{pH}-\mathrm{CaCl}_{2}$ de 5,5 , utilizado na citricultura brasileira, é definido a partir de correlações entre $\mathrm{V}$ na camada de $0-20 \mathrm{~cm}$ de profundidade e a produção de frutos de laranjeira (Quaggio et al., 2005). Entretanto, atualmente há controvérsias sobre a utilização do valor de $\mathrm{V}$ de $70 \%$, considerando-se inúmeros estudos de calagem em pomares de laranjeiras que não apresentaram aumento de produtividade em solos com $\mathrm{V}$ superior a 60 \% (Boaretto et al., 1996). Segundo esses autores, a calagem deveria ser recomendada quando o solo apresentasse V inferior a $40 \%$, elevando-a para $60 \%$.

A partir do início da produção da laranjeira ocorre exportação de nutrientes pelos frutos e, por conseguinte, diminuição dos teores de nutrientes no solo. No caso do Mg, é comum ocorrerem, nesse momento, sintomas foliares de deficiência nutricional, havendo a necessidade de reaplicações de calcário
(Fidalski \& Auler, 1997) para manter teores de Mg de $8 \mathrm{mmol}_{\mathrm{c}} \mathrm{dm}^{-3}$ nas entrelinhas do pomar na camada de $0-20 \mathrm{~cm}$ de profundidade (Quaggio et al., 2005). Em pomares estabelecidos, o suprimento de $\mathrm{Ca}$ e $\mathrm{Mg}$ deve ser obtido por meio de calagens superficiais, pois sua incorporação de calcário não é adequada por provocar a destruição de radicelas e raízes responsáveis pela nutrição das laranjeiras (Moreira, 1988) e favorecer a incidência de gomose (Oliveira et al., 1981).

Após o levantamento nutricional de pomares de laranjeira no noroeste do Paraná, no qual foi constatada deficiência nutricional por $\mathrm{Ca}$ e $\mathrm{Mg}$ devido à baixa freqüência de reaplicação de calcário (Fidalski \& Auler, 1997), têm sido feitas reaplicações anuais de calagem superficial com base nos resultados de análises químicas de solo na camada de $0-20 \mathrm{~cm}$ de profundidade. Esse procedimento foi importante para corrigir as deficiências nutricionais de $\mathrm{Mg}$ (Fidalski \& Auler, 1997) com a utilização de calcário dolomítico, que promoveu a redução das relações entre $\mathrm{Ca}$ e $\mathrm{Mg}$ no solo (Fidalski \& Tormena, 2005). Esses autores encontraram $\mathrm{V}$ de $64 \%$ na camada de $0-5 \mathrm{~cm}$ de profundidade, após três anos da distribuição superficial de calcário dolomítico nas entrelinhas de laranjeira "Folha Murcha" (Fidalski \& Tormena, 2005).

Com o uso contínuo de fertilizantes nitrogenados nas faixas de adubação adjacentes aos limites externos das projeções das copas das laranjeiras, e também com as maiores extrações de $\mathrm{Ca}$ e $\mathrm{Mg}$ pelas raízes, ocorre alteração química entre esta posição de amostragem de solo e o centro das entrelinhas (Fidalski \& Auler, 1997; Fidalski \& Stenzel, 2006), caracterizada pela maior acidificação e menor disponibilidade de Ca e $\mathrm{Mg}$ nas faixas de adubação. O gradiente de fertilidade química do solo entre as faixas de adubação e o centro 
das entrelinhas apresentou máxima produção de frutos da laranja "Valência", respectivamente, para valores de $\mathrm{V}$ de 30 e $60 \%$ na camada de $0-20 \mathrm{~cm}$ de profundidade (Quaggio et al., 2005).

O aumento da produção de laranja "Pêra" com a calagem superficial foi verificado por Vitti et al. (1992), após três calagens superficiais com intervalos de três e dois anos. As reaplicações de calcário em superfície com base no método de $\mathrm{V}$ para elevá-la a $70 \%$ na camada de $0-20 \mathrm{~cm}$ de profundidade podem estar sendo superdimensionadas, caracterizando a necessidade de avaliar a resposta da calagem superficial nessas duas posições de amostragem de solo.

O objetivo deste estudo foi quantificar as alterações químicas temporais nas faixas de adubação e entrelinhas, nutrição e produção de laranja "Pêra" após calagens superficiais, em um Argissolo Vermelho distrófico latossólico, no noroeste do Paraná.

\section{MATERIAL E MÉTODOS}

O estudo foi realizado em um experimento de laranjeira "Pêra" (Citrus sinensis) enxertada sobre o limoeiro "Cravo" (Citrus limonia) implantado em 1993 em uma área ocupada pela pastagem Brachiaria humidicola, no espaçamento de $4 \times 7 \mathrm{~m}$, na Fazenda São Judas Tadeu, localizada no Município de Alto Paraná, noroeste do Paraná ( $23^{\circ} 5$ ' S, $52^{\circ} 26$ ' W e altitude de $480 \mathrm{~m}$ ). O clima na região é subtropical (Cfa), sem estação seca definida e precipitações pluviais anuais de $1.500 \mathrm{~mm}$ (IAPAR, 2000). O solo foi identificado como Argissolo Vermelho distrófico latossólico textura arenosa/média A moderado e relevo suave ondulado (Embrapa, 2006). Os teores de argila e a espessura dos horizontes desse solo são os seguintes: A e AB (80 g kg-1 e 0-25 cm), Bt (180 g kg-1 e 26$55 \mathrm{~cm})$ e Bw $\left(210 \mathrm{~g} \mathrm{~kg}^{-1}\right.$ e $\left.56-200 \mathrm{~cm}\right)$, conforme Fidalski et al. (2007).

O delineamento experimental utilizado foi de blocos ao acaso, com três repetições e seis tratamentos de manejo das entrelinhas. Cada parcela experimental apresentava três linhas com cinco laranjeiras, com a parcela útil correspondente a três laranjeiras centrais $\left(84 \mathrm{~m}^{2}\right)$.

Em 1993, distribuiu-se sobre a gramínea Brachiaria humidicola $2 \mathrm{t} \mathrm{ha}^{-1}$ para elevar V a $70 \%$ de calcário dolomítico, no qual um dos tratamentos consistiu na ausência do revolvimento do solo nas entrelinhas, realizado somente na faixa de $2 \mathrm{~m}$ de largura, destinado ao plantio das laranjeiras e denominado de "preparo em faixas", mantendo-se a gramínea remanescente nas entrelinhas do pomar (Auler et al., 2008). Nos outros cinco tratamentos foi realizado o preparo convencional do solo na camada de $0-20 \mathrm{~cm}$ de profundidade com uma gradagem pesada, uma aração profunda e uma gradagem niveladora, incorporando o calcário para implantação dos seguintes tratamentos em 1993: cultivo de abacaxi cultivar "Smooth Cayenne" entre 1993-1995 em fileira dupla com adubação mineral ( $100 \mathrm{~g}$ de $\mathrm{P}_{2} \mathrm{O}_{5}, 30 \mathrm{~g}$ de $\mathrm{K}_{2} \mathrm{O}, 5 \mathrm{~g}$ de $\mathrm{Zn}$ e $2 \mathrm{~g}$ de $\mathrm{B}$ ), seguida da vegetação espontânea dessecada anualmente com herbicida pósemergente (Glyphosate) entre 1998 e 2000; semeadura da leguminosa Calopogonium mucronoides; semeadura da leguminosa amendoim forrageiro Arachis pintoi; plantio de mudas de Paspalum notatum; e a gramínea Brachiaria humidicula restabelecida espontaneamente após o preparo convencional do solo. A vegetação nas entrelinhas foi controlada com roçadas mecânicas conforme a necessidade, à exceção das leguminosas. Nas faixas de adubação, a vegetação foi controlada com capinas manuais e aplicação de herbicida pós-emergente (Glyphosate).

Anualmente foram realizadas adubações de cobertura com doses máximas de $165 \mathrm{~kg} \mathrm{ha}^{-1}$ de N, $70 \mathrm{~kg} \mathrm{ha}^{-1}$ de $\mathrm{P}_{2} \mathrm{O}_{5}$ e $120 \mathrm{~kg} \mathrm{ha}^{-1}$ de $\mathrm{K}_{2} \mathrm{O}$, nas formas de sulfato de amônio, superfosfato simples e cloreto de potássio, respectivamente; as adubações foliares foram realizadas para os nutrientes $\mathrm{B}$ e $\mathrm{Zn}$, com base nos resultados anuais das análises de solo e de tecido foliar.

Devido à necessidade de correção da acidez, da baixa disponibilidade de $\mathrm{Ca}^{2+} \mathrm{e} \mathrm{Mg}^{2+}$ no solo, do teor baixo de Ca e Mg nas folhas das laranjeiras e dos sintomas visuais típicos de deficiência por $\mathrm{Mg}$, no período de 1996 a 1999, foram distribuídas manualmente na superfície do solo das faixas de adubação e centro das entrelinhas, sem incorporação mecânica, as dosagens de calcário dolomítico e calcítico (Quadro 1). A determinação da necessidade dessas calagens foi definida pelo método de V para elevá-la a $70 \%$, baseando-se nos resultados das amostras de solo coletadas anualmente a $0-20 \mathrm{~cm}$ de profundidade. Entre 1997 e 1998, a utilização de calcário dolomítico e calcítico para elevar as proporções de $55 \%$ de Ca e $15 \%$ de Mg da capacidade de troca de cátions, para

Quadro 1. Fontes e doses de calcário equivalentes ao Poder Relativo de Neutralização Total (100 \%), distribuídos na superfície do solo das faixas de adubação e entrelinhas do pomar de laranjeira "Pêra", entre 1996 e 1999

Fonte de calcário $1996 \quad 1997 \quad 1998 \quad 1999 \quad$ Total

\begin{tabular}{llllll}
\hline & \multicolumn{5}{c}{ Faixas de adubação } \\
\cline { 2 - 6 } & \multicolumn{5}{c}{$\mathrm{ha}^{-1}$} \\
Dolomítico & 1,35 & 0,75 & 1,33 & 2,00 & 5,43 \\
Calcítico & 2,60 & 1,20 & 2,20 & 2,00 & 8,00 \\
& \multicolumn{5}{c}{ Entrelinhas } \\
Dolomítico & 0,00 & 2,00 & 0,39 & 2,00 & 4,39 \\
Calcítico & 0,00 & 0,00 & 2,20 & 2,00 & 4,20 \\
\hline
\end{tabular}


elevar V a 70 \%. A suspensão das reaplicações das calagens superficiais a partir de 2000 foi resultante da constatação das alterações químicas do solo pelas análises realizadas em maio de 1999.

A coleta de amostras de solo e a avaliação da produção de frutos foram realizadas anualmente no período de 1996 a 2004.

A amostragem de tecido foliar seguiu a recomendação da GPACC (1994), sendo coletadas entre janeiro e abril, a terceira e a quarta folha de ramos com frutos verdes nas extremidades, geradas na primavera com 6 a 8 meses de idade, na altura mediana e nos quatro quadrantes da copa das plantas laranjeiras (GPACC, 1994). As extrações de Ca e Mg foram feitas com a solução $\mathrm{HCl} 1 \mathrm{~mol} \mathrm{~L}^{-1}$, e as determinações analíticas com espectrometria de absorção atômica.

Coletaram-se amostras de solo em maio ou junho, nas faixas de adubação adjacente ao limite externo das projeções da copa das laranjeiras (distante 1,5 a $2,5 \mathrm{~m}$ do tronco das laranjeiras) e no centro das entrelinhas a 0-20 e 20-40 cm de profundidade, dos dois lados das laranjeiras, constituídas de seis subamostras de solo para as três laranjeiras úteis. Os resultados das análises químicas do solo, em 1996, caracterizaram a condição química do solo sem calagem (1994-1995). Foram realizadas as extrações de $\mathrm{Ca}^{2+}, \mathrm{Mg}^{2+} \mathrm{e} \mathrm{Al}^{3+}(\mathrm{KCl} ; 1$ mol L-1), K (Mehlich-1), $\mathrm{pH}-\mathrm{CaCl}_{2}\left(0,01 \mathrm{~mol} \mathrm{~L}^{-1}\right)$ e $\mathrm{H}+\mathrm{Al}$ (solução-tampão SMP). Com esses resultados, foram calculadas a CTC, a saturação por $\mathrm{Al}^{3+} \mathrm{e} \mathrm{V}$.

As avaliações da produção de laranja foram realizadas entre junho e setembro de 1996 a 2004, por meio da pesagem de frutos de três plantas em cada uma das parcelas experimentais. Nos anos agrícolas de 2000 e 2001 também foram computadas as produções colhidas em dezembro, originadas de segunda florada.

Para os conjuntos de 18 dados (seis tratamentos e três repetições) de cada um dos nove anos (1996 a 2004), dos resultados das análises químicas de solo (faixas de adubação e centro das entrelinhas nas camadas de $0-20$ e $20-40 \mathrm{~cm}$ de profundidade), dos teores foliares e da produção de laranja, foram calculados os intervalos de confiança da média:

$$
\mathrm{IC}=\overline{\mathrm{X}} \pm \mathrm{t}_{[(\mathrm{n}-1)(1-\alpha / 2)]} \frac{\mathrm{s}}{\sqrt{\mathrm{n}}}
$$

em que IC é o intervalo de confiança da média; $\overline{\mathrm{X}}$, a média aritmética; $t$, o valor da distribuição normal tabulado, obtido pelo número de dados (n) menos um grau de liberdade (n-1) e o grau de confiança para $95 \%(\alpha=0,05)$; e s, o desvio-padrão das amostras (Schlotzhauer \& Littell, 1997). Os intervalos de confiança da média das variáveis foram plotados graficamente em função de cada ano de avaliação, permitindo a comparação entre as posições de amostragem (faixas de adubação e centro das entrelinhas) e profundidades (0-20 e 20-40 cm). As características químicas do solo foram correlacionadas com a produção de frutos, por meio das correlações simples de Pearson e da significância dos coeficientes de correlações obtida pelo teste t.

\section{RESULTADOS E DISCUSSÃO}

As calagens superficiais realizadas entre $1996 \mathrm{e}$ 1999 aumentaram o $\mathrm{pH}-\mathrm{CaCl}_{2}$ a partir de 2000 na camada de $0-20 \mathrm{~cm}$ de profundidade, com valores máximos de 4,4 nas faixas de adubação e 5,9 no centro das entrelinhas (Figura 1). Em 2001, na mesma camada de solo, observaram-se valores máximos de $\mathrm{pH}-\mathrm{CaCl}_{2}$ de 4,9 e 6,4 nas faixas de adubação e no centro das entrelinhas, respectivamente. $\mathrm{O}$ aumento do $\mathrm{pH}-\mathrm{CaCl}_{2}$ promovido pelas calagens superficiais precipitou o $\mathrm{Al}^{3+}$ na profundidade de $0-20 \mathrm{~cm}$, com conseqüente redução na saturação por $\mathrm{Al}^{3+}$ (Figura 1). Maiores valores de $\mathrm{pH}-\mathrm{CaCl}_{2}$ nas entrelinhas do que nas faixas de adubação de pomares de laranjeira após calagens superficiais foram obtidos por Vitti et al. (1992). O gradiente de acidez entre as posições de amostragem de solo indica a necessidade de reaplicação de calagem superficial nas faixas de adubação dos pomares de laranjeira em conseqüência da acidificação do solo pelo emprego da adubação nitrogenada (Fidalski \& Auler, 1997; Fidalski \& Stenzel, 2006).

A redução da acidez do solo e o suprimento de $\mathrm{Ca}$ com as calagens superficiais refletiram na maior disponibilidade deste elemento a partir de 2000, na camada de $0-20 \mathrm{~cm}$ de profundidade no centro das entrelinhas e nas faixas de adubação (Figura 2). Os valores máximos de $14,7 \mathrm{mmol}_{\mathrm{c}} \mathrm{dm}^{-3}(34 \%$ da CTC) e $21,5 \mathrm{mmol}_{\mathrm{c}} \mathrm{dm}^{-3}$ (46\% da CTC) de $\mathrm{Ca}^{2+}$ ocorreram na camada de 0-20 cm de profundidade em 2001, nas faixas de adubação e no centro das entrelinhas, respectivamente. Comparando esses resultados com o valor referencial de se manter $40 \%$ da CTC com $\mathrm{Ca}^{2+}$ em citros (GPACC, 1994), somente o centro das entrelinhas, na camada de $0-20 \mathrm{~cm}$ de profundidade, atenderia esse índice mínimo em 2001 e 2002 (Figura 2). Calagens superficiais em citros mostraram também aumento dos teores de $\mathrm{Ca}^{2+}$ nas faixas de adubação a 0-20 $\mathrm{cm}$ de profundidade (Fidalski \& Stenzel, 2006).

A partir de 2001 houve aumentos dos teores de $\mathrm{Mg}^{2+}$ na profundidade de $0-20 \mathrm{~cm}$, diferenciando-se estatisticamente em 2001 da condição original (1996), observando-se em 2004 valores máximos de $6,5 \mathrm{mmol}_{\mathrm{c}} \mathrm{dm}^{-3}(14 \%$ da CTC) nas faixas de adubação de $10,4 \mathrm{mmol}_{\mathrm{c}} \mathrm{dm}^{-3}$ (22\% da CTC) no centro das entrelinhas. As calagens superficiais mantiveram o índice mínimo de $10 \%$ da proporção de $\mathrm{Mg}^{2+}$ na CTC recomendado para os citros (GPACC, 1994) ou de $8 \mathrm{mmol}_{\mathrm{c}} \mathrm{dm}^{-3}$ no centro das entrelinhas a $0-20 \mathrm{~cm}$ de profundidade (Quaggio et al., 1992), os quais também 

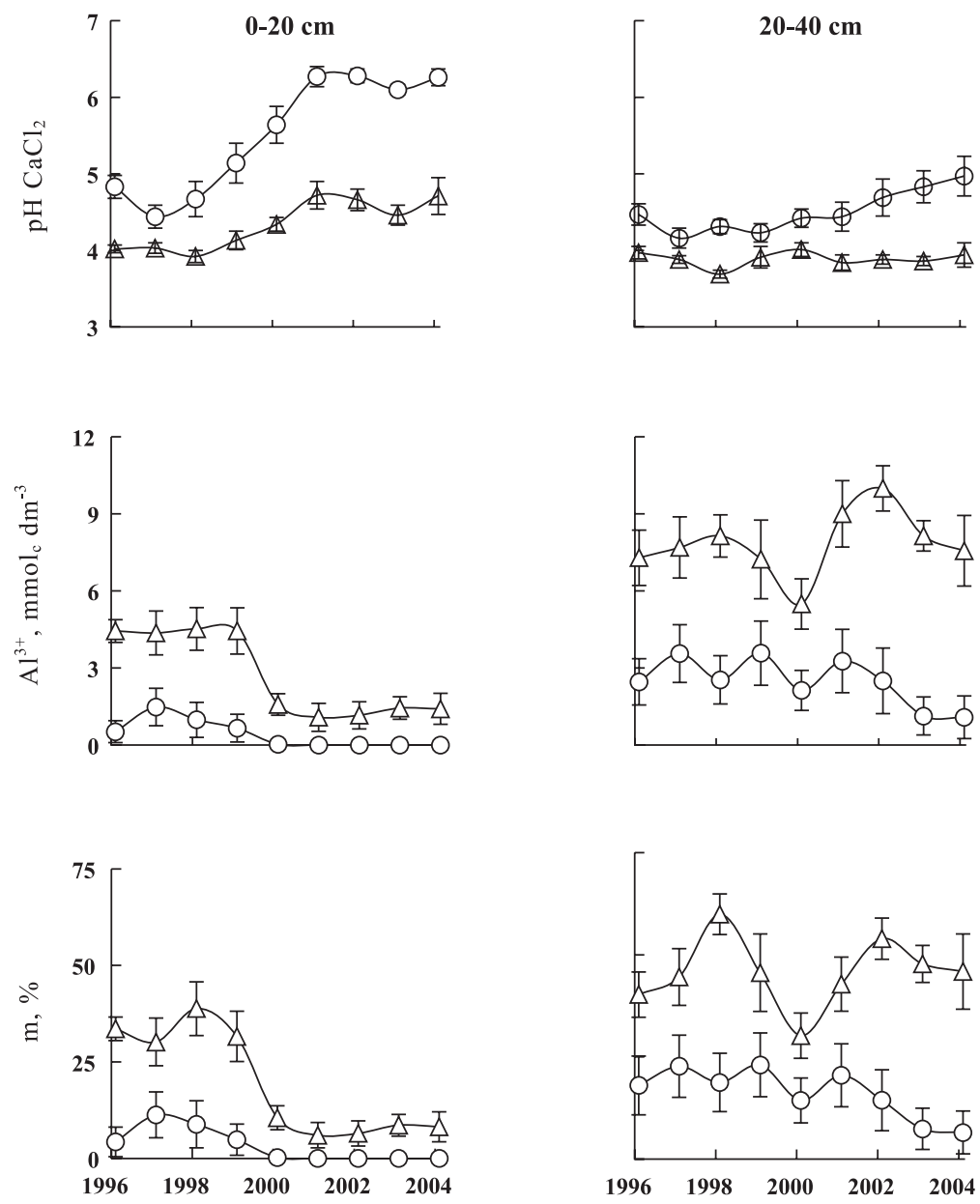

ANO

Figura 1. Valores médios e intervalos de confiança da média $(\mathrm{p}<0,05)$ pelo teste $\mathrm{t}(\mathrm{barras}$ verticais) do $\mathrm{pH}-$ $\mathrm{CaCl}_{2}, \mathrm{Al}^{3+}$ e saturação por $\mathrm{Al}^{3+}(\mathrm{m})$, antes das calagens superficiais (1996) e após as calagens superficiais (1997-1999), nas faixas de adubação $(\Delta)$ e no centro das entrelinhas $(O)$ a 0-20 e 20-40 cm de profundidade.

foram obtidos por Fidalski \& Stenzel (2006), com o mesmo número e período de reaplicações superficiais de calcário dolomítico em laranjeira "Folha Murcha", em solo semelhante ao do presente estudo.

Os valores de V (Figura 3) apresentaram comportamento semelhante ao dos valores de $\mathrm{pH}-\mathrm{CaCl}_{2}$ e aos teores de $\mathrm{Ca}^{2+}$ na camada de 0-20 $\mathrm{cm}$ de profundidade (Figuras 1 e 2). Nessa camada, em relação à condição original com $\mathrm{V}$ máxima de $38 \%$ no centro das entrelinhas (1996), as calagens superficiais realizadas em 1996-1999 elevaram a V para $54 \%$ em 2000, com valor máximo de 64 \% em 2001 (Figura 3) valores próximos da $\mathrm{V}$ utilizada como índice de referência para a calagem em pomares de laranjeiras (GPACC, 1994; Boaretto et al., 1996; Quaggio et al., 2005). Nas faixas de adubação ocorreu proporcionalmente a mesma alteração da $\mathrm{V}$ verificada no centro das entrelinhas, porém em menor magnitude, atingindo valor máximo de 46 \% em 2001 (Figura 3).
Na camada de 20-40 cm de profundidade houve alteração do valor de $\mathrm{pH}-\mathrm{CaCl}_{2}$ no centro das entrelinhas observadas a partir de 2003, com valor máximo de 5,0 (Figura 1). Contudo, nas faixas de adubação, a calagem não alterou o pH-CaCl $\mathrm{Cl}_{2} \mathrm{e}$ manteve alta disponibilidade de $\mathrm{Al}^{3+}$ nas faixas de adubação em $2002\left(10,3 \mathrm{mmol}_{\mathrm{c}} \mathrm{dm}^{-3} ; 59 \%\right.$ da saturação por $\mathrm{Al}^{3+}$ ). A disponibilidade de $\mathrm{Ca}^{2+}$ na camada de 20-40 cm aumentou significativamente somente em 2004 (Figura 2), nas faixas de adubação com valores máximos de $3,6 \mathrm{mmol}_{\mathrm{c}} \mathrm{dm}^{-3}$ (8 \% da CTC) e no centro das entrelinhas com teores máximos de $8,2 \mathrm{mmol}_{\mathrm{c}} \mathrm{dm}^{-3}$ (18\% da CTC). Essas alterações químicas do solo aumentaram a $\mathrm{V}$ para valor máximo de $24 \%$ nas faixas de adubação e de $46 \%$ no centro das entrelinhas em 2004 (Figura 3). Esses resultados mostram a ineficiência da calagem superficial utilizada na neutralização dessa barreira química formada na camada de $20-40 \mathrm{~cm}$ sob a faixa de 
adubação, cujos valores de saturação por $\mathrm{Al}^{3+}$ caracterizam caráter álico nos períodos de 1997-1999 e 2002-2004 (Figura 1).

Os máximos teores de Ca nas folhas de laranjeiras foram de 35-38 $\mathrm{g} \mathrm{kg}^{-1}$ (Figura 4), à exceção de $1998 \mathrm{e}$ 2000, que se mantiveram semelhantes à condição original de 1996 (28-31 $\left.\mathrm{g} \mathrm{kg}^{-1}\right)$. A absorção e a acumulação de Ca pelas folhas de laranjeira se estabilizaram a partir de 2001, coincidindo com a máxima disponibilidade de $\mathrm{Ca}^{2+}$ no solo (Figura 2). Os intervalos de confiança das médias dos teores de Ca pertencem ao teor adequado de 35-45 $\mathrm{g} \mathrm{kg}^{-1}$ sugerido por Quaggio et al. (2005), com exceção de 1996, 1998 e 2000 (Figura 4). Os teores foliares de Mg também variaram entre 1996 e 2000, aumentando a partir de 2001 (Figura 4), proporcionalmente aos aumentos de $\mathrm{Mg}^{2+}$ no solo (Figura 2). Os teores de $\mathrm{Mg}$ correspondem ao nível baixo, com teores inferiores a $3 \mathrm{~g} \mathrm{~kg}^{-1}$ (Quaggio et al., 2005), mesmo tendo havido teores deste nutriente no solo a partir de 2002 de $8 \mathrm{mmol}_{\mathrm{c}} \mathrm{dm}^{-3}$ na camada de 0-20 $\mathrm{cm}$ de profundidade na entrelinha do pomar (Figura 2), necessários para a produção de laranja (Quaggio et al., 1992). A estabilidade nutricional quanto ao Ca nas folhas das laranjeiras ocorreu a partir de 2001, com a máxima reação do calcário na camada de $0-20 \mathrm{~cm}$ de profundidade (Figuras 1, 2 e 3), satisfazendo as necessidades nutricionais das laranjeiras por $\mathrm{Ca}$, porém não com o $\mathrm{Mg}$, atribuído ao comportamento das laranjeiras de priorizar a absorção de $\mathrm{Ca}$ em detrimento do $\mathrm{Mg}$ (Fidalski \& Stenzel, 2006).
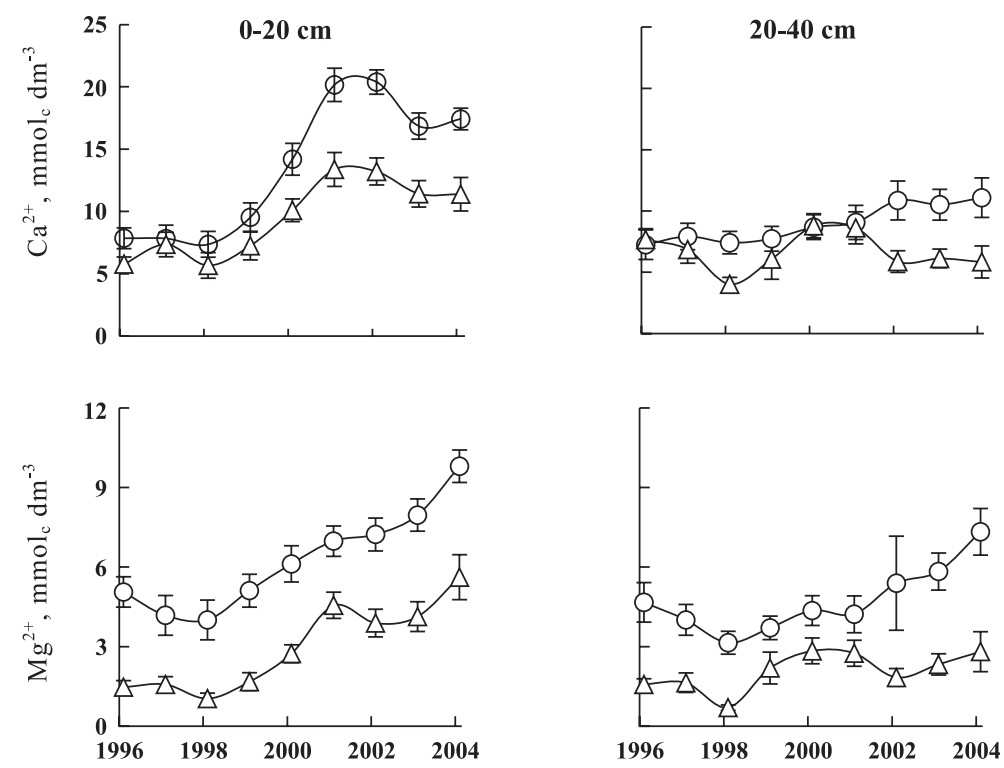

ANO

Figura 2. Teores médios e intervalos de confiança da média $(\mathrm{p}<0,05)$ pelo teste $\mathrm{t}$ (barras verticais) de $\mathrm{Ca}^{2+}$ e $\mathrm{Mg}^{2+}$, antes (1996) e após as calagens superficiais (1997-1999), nas faixas de adubação ( $\triangle$ ) e no centro das entrelinhas (O) a 0-20 e 20-40 cm de profundidade.
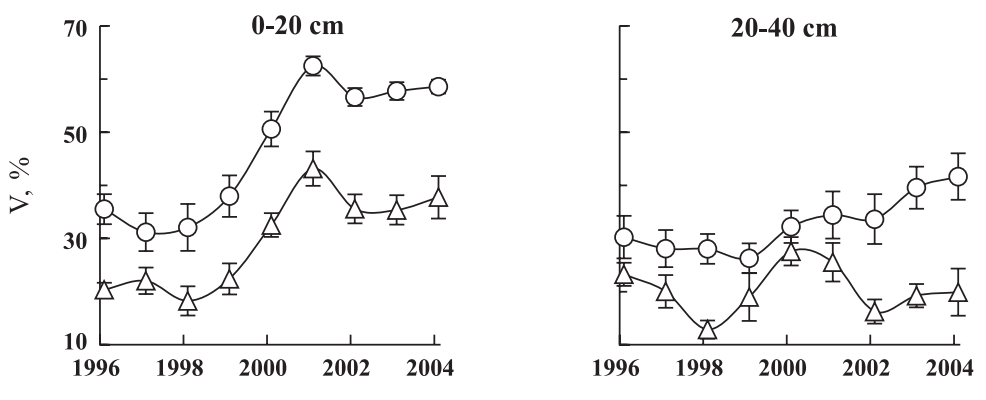

ANO

Figura 3. Valores médios e intervalos de confiança da média $(\mathrm{p}<\mathbf{0 , 0 5})$ pelo teste $\mathrm{t}(\mathrm{barras}$ verticais) da saturação por bases (V), antes (1996) e após as calagens superficiais (1997-1999), nas faixas de adubação $(\triangle)$ e no centro das entrelinhas $(O)$ a 0-20 e 20-40 cm de profundidade. 
A produção de frutos de laranja "Pêra" aumentou entre 1997 e 2004 em relação à condição original de 1996, na qual os frutos haviam se desenvolvido sem a calagem superficial em 1995/1996 (Figura 5). A máxima produção de frutos verificada em 2000 coincidiu com a melhoria da fertilidade química do solo constatada pela menor acidez, maior disponibilidade de cátions e maior $\mathrm{V}$ e estado nutricional das laranjeiras (Figuras 1, 2, 3 e 4). A redução da produção de frutos de laranja em 2001 foi causada pelas condições climáticas, que comprometeram a produção de laranja no noroeste do Paraná, o que também foi observado na laranjeira "Folha Murcha" sobre diferentes porta-enxertos por Stenzel et al. (2005).


Figura 4. Teores médios e intervalos de confiança da média $(p<0,05)$ pelo teste $t$ (barras verticais) de Ca e Mg nas folhas das laranjeiras, antes (1996) e após as calagens superficiais (1997-1999).
Houve correlação significativa entre a produção de frutos de laranjeira "Pêra" e as características químicas do solo: positiva com $\mathrm{pH}-\mathrm{CaCl}_{2}, \mathrm{Ca}^{2+}, \mathrm{Mg}^{2+}$ e $\mathrm{V}$ e negativa com $\mathrm{Al}^{3+}$ (Quadro 2). A exclusão dos dados de produção de frutos de laranja de 2001 explicou mais de 59 \% do grau de dependência da produção de frutos com características químicas do solo nas faixas de adubação e no centro das entrelinhas a 0-20 cm de profundidade, à exceção dos teores de $\mathrm{Al}^{3+}$ no centro das entrelinhas, enquanto na profundidade de 20 $40 \mathrm{~cm}$ os coeficientes explicaram no máximo $46 \%$ (Quadro 2). Os resultados dessas correlações indicam a dependência das laranjeiras à menor acidez e à maior

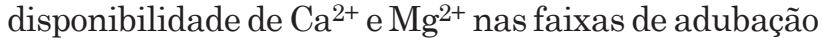
e no centro das entrelinhas, principalmente na camada de 0-20 cm de profundidade (Figuras 1, 2, 3 e 5; Quadro 2).

Os resultados deste estudo reiteram as sugestões do manejo da calagem superficial em solos desenvolvidos a partir do arenito da Formação Caiuá (Fidalski \& Auler, 1997), mantendo-se a qualidade deste solo com a vegetação permanente nas entrelinhas (Fidalski et al., 2007), indispensável para o seu manejo

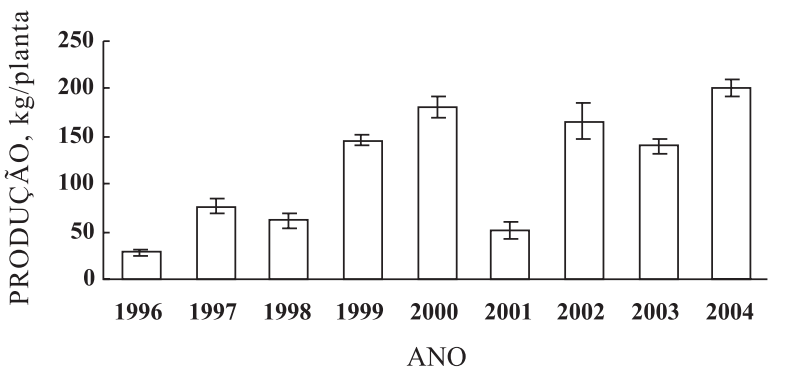

Figura 5. Valores médios e intervalos de confiança da média $(\mathrm{p}<0,05)$ pelo teste $t$ (barras verticais) da produção de laranja "Pêra" no período experimental (1996-2004).

Quadro 2. Coeficientes de correlações lineares simples de Pearson entre a produção de frutos da laranjeira "Pêra" com as variáveis do solo, nas faixas de adubação e centro das entrelinhas, a 0-20 e 20-40 cm de profundidade

\begin{tabular}{|c|c|c|c|c|c|c|c|c|}
\hline \multirow{3}{*}{$\begin{array}{c}\text { Característica } \\
\text { química }\end{array}$} & \multicolumn{4}{|c|}{ Faixas de adubação } & \multicolumn{4}{|c|}{ Centro das entrelinhas } \\
\hline & \multicolumn{2}{|c|}{$0-20 \mathrm{~cm}$} & \multicolumn{2}{|c|}{$20-40 \mathrm{~cm}$} & \multicolumn{2}{|c|}{$0-20 \mathrm{~cm}$} & \multicolumn{2}{|c|}{$20-40 \mathrm{~cm}$} \\
\hline & $\mathrm{n}=162^{(1)}$ & $\mathrm{n}=144^{(2)}$ & $\mathrm{n}=162^{(1)}$ & $\mathrm{n}=144^{(2)}$ & $\mathrm{n}=162^{(1)}$ & $\mathrm{n}=144^{(2)}$ & $\mathrm{n}=162^{(1)}$ & $\mathrm{n}=144^{(2)}$ \\
\hline $\mathrm{pH}-\mathrm{CaCl}_{2}$ & $0,40 * *$ & $0,63 * *$ & $0,17^{*}$ & $0,15 \mathrm{~ns}$ & $0,49 * *$ & $0,71^{* *}$ & $0,35^{* *}$ & $0,37 * *$ \\
\hline $\mathrm{Al}^{3+}$ & $-0,43 * *$ & $-0,61 * *$ & $-0,08 \mathrm{~ns}$ & $-0,02 \mathrm{~ns}$ & $-0,26 * *$ & $-0,34 * *$ & $-0,23 * *$ & $-0,21 * *$ \\
\hline $\mathrm{Ca}^{2+}$ & $0,40 * *$ & $0,66^{* *}$ & $-0,06 \mathrm{~ns}$ & $0,05 \mathrm{~ns}$ & $0,41^{* *}$ & $0,69 * *$ & $0,34 * *$ & $0,40 * *$ \\
\hline $\mathrm{Mg}^{2+}$ & $0,46 * *$ & $0,66 * *$ & $0,32^{* *}$ & $0,46 * *$ & $0,50 * *$ & $0,59 * *$ & $0,37 * *$ & $0,38 * *$ \\
\hline $\mathrm{V}^{(3)}$ & $0,37 * *$ & $0,68 * *$ & $-0,01 \mathrm{~ns}$ & $0,10 \mathrm{~ns}$ & $0,42^{* *}$ & $0,69 * *$ & $0,28 * *$ & $0,36 * *$ \\
\hline
\end{tabular}

(1) Nove colheitas (1996-2004). ${ }^{(2)}$ Oito colheitas (1996-2000 e 2002-2004). ${ }^{* *}(\mathrm{p}<0,01),{ }^{*}(\mathrm{p}<0,05)$ e ns $(\mathrm{p}>0,05) .{ }^{(3)}$ Saturação por bases. 
sem revolvimento mecânico. Ao mesmo tempo, os resultados indicam a necessidade prévia de quatro anos para obter as máximas alterações na camada de 0-20 cm de profundidade, limitando a expectativa da V em $45 \%$ nas faixas de adubação (Figuras 1, 2, 3 e 4) - tempo considerado adequado para uma cultura permanente com expectativa de vida útil mínima de 15 anos.

\section{CONCLUSÕES}

1. As máximas alterações das características químicas do solo na camada de $0-20 \mathrm{~cm}$ de profundidade ocorreram em 2001, após as calagens superficiais realizadas entre 1996 e 1999.

2. Teores adequados de Ca nas folhas das laranjeiras também foram observados a partir de 2001 na camada de 0-20 $\mathrm{cm}$ de profundidade.

3. A produção de laranja correlacionou-se negativamente com $\mathrm{Al}^{3+}$ e positivamente com $\mathrm{pH}-\mathrm{CaCl}_{2}, \mathrm{Ca}^{2+}$

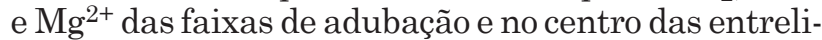
nhas.

4. As calagens superficiais elevaram a saturação por bases (V) na camada de 0-20 $\mathrm{cm}$ de profundidade até 46 e $64 \%$ das faixas de adubação e entrelinhas, respectivamente.

\section{LITERATURA CITADA}

AULER, P.A.M.; FIDALSKI, J.; PAVAN, M.A. \& NEVES, C.S.V.J. Produção de Laranja 'Pêra' em sistemas de preparo de solo e manejo nas entrelinhas. R. Bras. Ci. Solo, 32:363-374, 2008 .

BOARETTO, A.E.; MURAOKA, T. \& RÊGO, I.C. Calagem e gessagem em citricultura. In: SEMINÁRIO INTERNACIONAL DE CITROS, 4., 1996, Bebedouro. Anais. Campinas, Fundação Cargill, 1996. p.115-129.

EMPRESA BRASILEIRA DE PESQUISA AGROPECUÁRIA . EMBRAPA. Sistema brasileiro de classificação de solos. 2.ed. Rio de Janeiro, Embrapa Solos, 2006. 306p.

FIDALSKI, J. \& AULER, P.A.M. Levantamento nutricional de pomares de laranja no noroeste do Paraná. Arq. Biol. Tecnol., 40:443-451, 1997.

FIDALSKI, J. \& STENZEL, N.M.C. Nutrição e produção da laranjeira "Folha Murcha" em porta-enxertos e plantas de cobertura permanente na entrelinha. Ci. Rural, 36:807$813,2006$.
FIDALSKI, J. \& TORMENA, C.A. Dinâmica da calagem superficial em um Latossolo Vermelho distrófico. R. Bras. Ci. Solo, 29:235-247, 2005.

FIDALSKI, J.; TORMENA, C.A. \& SILVA, A.P. Qualidade física do solo em pomar de laranjeira no noroeste do Paraná com manejo da cobertura permanente na entrelinha. R. Bras. Ci. Solo, 31:423-433, 2007.

GRUPO PAULISTA DE ADUBAÇÃO E CALAGEM PARA CITROS - GPACC. Recomendações de adubação e calagem para citros no Estado de São Paulo. 3.ed. Laranja, Edição Especial, p.1-27, 1994

INSTITUTO AGRONÔMICO DO PARANÁ - IAPAR. Cartas climáticas do Paraná: edição ano 2000. versão 1.0. Londrina, 2000. CD-ROM.

MATTOS JR., D.; QUAGGIO, J.A.; CANTARELLA, H. \& ALVA, A.K. Nutrient content of biomass components of Hamlin sweet orange trees. Sci. Agric., 60:155-160, 2003.

MOREIRA, C.S. Manejo do solo em pomar cítrico. Laranja, 9:423-435, 1988.

OLIVEIRA, A.A.R.; SANTOS FILHO, H.P.; COELHO, Y.S.; PASSOS, O.S. \& CUNHA SOBRINHO, A.P. Relação entre manejo do solo e ocorrência de gomose de Phytophthora spp. em pomar de laranja 'Baianinha' (Citrus sinensis (L.) Osbeck). Pesq. Agropec., Bras.,16:841-844, 1981.

PAVAN, M.A. \& JACOMINO, A.P. Root growth and nutrient contents of citrus rootstocks in an acid soil with varied pH. Ci. Cult., 50:56-59, 1998 .

QUAGGIO, J.A.; MATTOS JUNIOR, D. \& CANTARELLA, H. Manejo da fertilidade do solo na citricultura. In: MATTOS JUNIOR, D.; DE NEGRI, J.D.; PIO, R.M. \& POMPEU JUNIOR., J., orgs. Citros. Campinas, Instituto Agronômico, 2005. v.1. p.483-517.

QUAGGIO, J.A.; TEÓFILO SOBRINHO, J. \& DECHEN, A.R. Magnesium influences on fruit yield and quality of 'Valencia' sweet orange on 'Rangpur' lime. In: INTERNATIONAL CITRUS CONGRESS, 7., Acireale, 1992. Proceedings. Acireale, 1992. v.2. p.633-637.

SCHLOTZHAUER, S.R. \& LITTELL, R.C. SAS ${ }^{\circledR}$ System for elementary statistical analysis. 2.ed. Cary, SAS Institute, 1997. 456p.

STENZEL, N.M.C.; NEVES, C.S.V.J.; SCHOLZ, M.B.S. \& GOMES, J.C. Comportamento da laranjeira 'Folha Murcha' em sete porta-enxertos no noroeste do Paraná. R. Bras. Frutic., 27:408-411, 2005.

VITTI, G.C.; DONADIO, L.C.; MALAVOLTA, E. \& CABRITA J.R.M. Effects of lime and phosphogypsum on citrus. In: INTERNATIONAL COLLOQUIUM FOR THE OPTIMIZATION OF PLANT NUTRITION, 8., Lisboa, 1992. Optimization of plant nutrition. Lisboa, 1992. p.449452. 